\title{
Expressional Comparison Between Episomal and Stable Transfection of a Selected tri-fusion Protein in Leishmania tarentolae
}

\author{
Tahereh Taheri, Elham Gholami, Faeze Saatchi, Negar Seyed, Yasaman Taslimi, Sima Rafati* \\ Molecular Immunology and Vaccine Research Laboratory, Pasteur Institute of Iran, Tehran, Iran
}

Received May 6, 2014; Accepted May 18, 2014

\begin{abstract}
:
Introduction: Leishmania tarentolae (L. tarentolae) is a nonpathogenic species of Leishmania genus that can be used as an expression system to produce immunogenic proteins or epitopes in vivo acting as an efficient and safe recombinant live vector in vaccinology. Cysteine proteases (CPs) are one group of Family C1 peptidases in Leishmania that are important for survival of the parasite within the host and are introduced as potential vaccine candidates. Two types of cysteine proteases, CPA and CPB, play a critical role in Leishmania. Previously, it has indicated that immunization with two genes or recombinant proteins of $\mathrm{CPA}$ and $\mathrm{CPB}$ individually or fused together with various adjuvant or combined with liposome are able to elicit a protective immune response against $L$ major in BALB/c mice. Methods: In this study, for the first time, two lines of recombinant non-pathogenic Leishmania were generated by transfection of a heterologous triple fusion gene encoding $c p a / c p b /$ egfp. For this purpose, two different expressions approaches were taken; episomal expression using rDNA promoter, and integrative expression from rRNA locus of genome. Results: After genotype confirmation, the fluorescence intensity was monitored in different phases of parasite growth by both fluorescence microscopy and FACS analysis.Western blot and RT-PCR analysis showed that $c p a / c p b / e g f p$ tri-fused genes were specifically expressed in both lines of transgenic parasites. Conclusion: In this study a single fused gene was expressed in both systems and the results showed that the expression of integrated gene was higher than episomal. In addition, the present data suggest that $L$. tarentolae expressing $c p a / c p b /$ egfp is a promising carrier as vectored vaccine in future research. Vac Res, 2014, 1 (1): 1 - 9
\end{abstract}

Keywords: Leishmania tarentolae, Knock-in, Cysteine Protease, EGFP.

\section{INTRODUCTION}

Leishmaniasis is an infectious disease more prevalent in the developing countries, caused by Leishmania parasites and is still a serious problem in $21^{\text {st }}$ century [1]. Cutaneous and visceral leishmaniasis, the two main forms of this infection, have a long history in Iran. Due to several reasons, including toxicity, long-term duration, drug resistance, side effects of current drugs, antigenic variation and complexity of the life cycle of Leishmania, developing an efficient approach to control the infection is challenging [2]. Importantly, leishmaniasis is known as a tool-deficient disease since no accurate system for its detection is available. Vaccination with recombinant proteins is one of the best approaches for combating or controlling leishmaniasis and early attempts at production of recombinant proteins were focused on prokaryote expression systems such as E. coli. However, these expression systems are not the best choices due to the limitations in codon usage, post-translational modifications, or the formation of insoluble inclusion bodies and incorrect folding of the proteins [3]. On the other hand proteins expressed in eukaryotic expression hosts are post-translationally modified and these hosts are now commonly used for protein production [4].

Since 1990, using non-pathogenic or attenuated organisms as a novel approach for protein expression has attracted attention. L. tarentolae is a lizard parasite and non-pathogenic for humans that can be used for development of live vaccines [5]. Promastigote form of $L$. tarentolae is able to infect macrophages in vitro [6], and is increasingly being used as a

*Corresponding Author: Sima Rafati, Molecular Immunology and Vaccine Research Laboratory, Pasteur Institute of Iran, Tehran, Iran. No. 69, Pasteur Ave, Tehran, Iran, 1316943551.

Email: s_rafati@yahoo.com

Tel: +98 2166953311 Ext. 2112, $\quad$ Fax: +98 2166953311 Ext. 2115 
new eukaryotic expression system [7] and a safe and reliable live vector for mammalian vaccination [8].

CPA and CPB are members of the cysteine protease family with molecular weights of 27 and $40 \mathrm{kDa}$, encoded by cpa and cpb genes of approximately 700 and $950 \mathrm{bp}$, respectively. cpa is a single-copy gene and is highly expressed in both promastigote and amastigote phase, but $c p b$ is a multi-copy gene and is highly expressed only in amastigotes [9-10]. Furthermore, $c p b$ contains a short tail sequence in its C-terminal extension (CTE) that is not conserved within the family and is removed during processing and maturation [11-12] and has been shown that this region is highly immunogenic in mammalian host [12]. Interestingly, studies have shown that mutant L. mexicana ${ }^{\Delta \mathrm{cpa}-\mathrm{cpb}}$ parasites fail to produce lesion in mice and hamsters [13].

Discovery of reporter genes has enabled the investigators to follow the Leishmania in infected tissues or cells, to study gene function, to evaluate the effect of anti-leishmanial drugs in vitro and in vivo and monitor parasites maintenance in animals [14-15].

Among reporter genes, GFP (green fluorescent protein) is the most versatile and its expression (transiently or stably) can be detected using fluorescence microscopy or flow cytometry. There is a direct correlation between the number of a parasite and EGFP (enhanced GFP) fluorescence intensity [16]. Furthermore, studies on GFP-expressing Leishmania strains have shown that GFP could be used as a reporter gene to follow Leishmania in macrophages or tissues [17]. Although expressing proteins transitionally is faster and easier than stable expressions, it has some limitations such as variation in plasmid copy number from parasite to parasite and the instability of GFP fluorescence intensity during parasite growth and its reduction in the absence of selection markers pressure $[2,18]$. In many cases, it is beneficial and/ or essential to develop stable cell lines expressing the protein of interest. Integrated reporter genes in genome could be replicated along with endogenous genes and cell components without the need for keeping the parasites under selection pressures (such as antibiotics).

Here, we describe generation of recombinant $L$. tarentolae strains expressing $c p a / c p b / e g f p$ triple fusion gene integrated in rRNA locus and episomally using a plasmid vector. The level of protein expression of tri fused cpa/cpb/egfp in these strains is compared and discussed.

\section{MATERIALS AND METHODS}

Plasmid construction. DNA fragment containing $c p a / c p b / e g f p$ coding regions was prepared and cloned in pLEXSY-neo2 vector (Jena bioscience, Germany) as previously described and used to integrate the fused gene in the genome of the parasite [19]. In order to make an episomal expression vector for $c p a / c p b / e g f p$, this triple fused gene was digested from pEGFP-cpa/cpb/egfp using BamHI and NheI and cloned into BamHI and XbaI sites of the pSP72-L.m rDNA Pro- $\alpha$ IR- $\alpha$ IR-Neo vector (kindly provided by Prof. Barbara Papadopoulou, Laval University, Canada). To evaluate in vitro expression of recombinant proteins, COS-7 cells were transfected transiently with pEGFP-cpa/cpb/egfp plasmid using the PEI (Poly sciences, Europe) reagent as described previously [20]. Briefly, the mixture of $10 \mathrm{mM}$ PEI and 5 $\mu \mathrm{g}$ DNA in HBS buffer ( $\mathrm{NaCl} 0.15 \mathrm{M}$ and HEPES $0.02 \mathrm{M}$, $\mathrm{pH} 7.4$ ) was added to $2.5 \times 10^{5}$ cells seeded in 4 well plates (SPL life sciences) at $70 \%$ confluency in serum free medium (Gibco, Germany). Six hours later, cells were washed and further incubated at $37^{\circ} \mathrm{C}$ humidified incubator for at least $24 \mathrm{~h}$. The transfected cells harboring cpa/cpb/egfp as an episomal vector were monitored by fluorescence microscopy and fluorescent intensity at different time points was measured by flow cytometry.

Leishmania strains and culture conditions. Promastigotes of wild-type (WT) L. tarentolae Tar II (ATCC 30267) were cultured in vitro in M199 media (Sigma, Germany) supplemented with $5 \%$ heat-inactivated fetal calf serum (HI-FCS, Gibco, UK), $40 \mathrm{mM}$ HEPES, $2 \mathrm{mM}$ L-glutamine, $0.1 \mathrm{mM}$ adenosine, $0.5 \mu \mathrm{g} / \mathrm{ml}$ hemin and $50 \mu \mathrm{g} / \mathrm{ml}$ gentamicin (all from Sigma, Germany) at $26^{\circ} \mathrm{C}$ in aerobic conditions. Promastigotes in logarithmic phase were used for transfection and in stationary-phase to determine the in vitro macrophage infectivity rate. In all experiments, an egfp-integrated L. tarentolae strain was used as positive control and maintained in complete M199 media without antibiotics [17]. Episomally-transfected parasites were cultured in complete M199 media with at least $50 \mu \mathrm{g} / \mathrm{ml}$ of G418. Knock-in Leishmania was maintained in the absence or presence of different concentrations of G418 (Geneticin, Gibco, UK).

Transfection. Parasites in the late log growth phase were harvested by two step centrifugation. First step at $700 \mathrm{rpm}, 7$ min, $4^{\circ} \mathrm{C}$ precipitated dead cells, second step at $3000 \mathrm{rpm}, 10$ min, $4^{\circ} \mathrm{C}$ pelleted the live parasites. Cells were then washed with phosphate buffered saline (PBS) and resuspended in ice-cold electroporation buffer (21 mM HEPES, $137 \mathrm{mM}$ $\mathrm{NaCl}, 5 \mathrm{mM} \mathrm{KCl}, 0.7 \mathrm{mM} \mathrm{Na} 2 \mathrm{HPO} 4,6 \mathrm{mM}$ glucose; $\mathrm{pH} 7.5$ ) to a final density of 108 parasites $/ \mathrm{ml}$, as recommended [21]. Parasites $\left(4.0 \times 10^{7} /-300 \mu \mathrm{l}\right)$ were mixed with $20 \mu \mathrm{g}$ plasmid DNA in $0.2 \mathrm{~cm}$ electroporation cuvette (BioRad, USA) and kept on ice for $10 \mathrm{~min}$. Electroporation was performed twice at $450 \mathrm{~V}, 500 \mu \mathrm{F}$ using Bio Rad Gene Pulser Ecell (Bio-Rad, USA) and cell suspension was immediately put on ice and kept for $10 \mathrm{~min}$. Electroporated parasites were then transferred to $3 \mathrm{ml}$ complete M199 media supplemented with $10 \%$ FBS without antibiotic and incubated at $26^{\circ} \mathrm{C}$ for $24 \mathrm{~h}$. To select transient transfectants, electroporated parasites were cultured in liquid M199 media containing $10 \mu \mathrm{g} / \mathrm{ml} \mathrm{G} 418$ and incubated at $26^{\circ} \mathrm{C}$ for $48 \mathrm{~h}$. Concentration of G418 in media was gradually increased during each passages, motility and viability of parasites were checked under an inverted microscope. Wild-type L. tarentolae was electroporated with no insert as control. EGFP expression in cells was monitored after each passage by an epifluorescence microscope. One of the clones which expressed both EGFP and CPA/CPB proteins was chosen for the subsequent studies.

Genotype confirmation of transfected parasites. Genomic DNA from parasites was isolated using GF-1 Nucleic Acid Extraction kit (Vivantis, USA) and used for DNA hybridization and PCR. For Southern blotting, the genomic DNA was digested with $\mathrm{BamHI}$ and separated on $0.8 \%$ agarose gel and electrotransferred to nylon membrane (Boehringer Mannheim, Germany) according to the standard protocol. 
The egfp ORF (100 ng) was labeled with radiolabeled dCTP in PCR reaction and after purification used as a probe for hybridization. The membrane was washed three times, first with $2 \mathrm{X} \mathrm{SSC}, 0.5 \% \mathrm{SDS}$ at $25^{\circ} \mathrm{C}$ for $30 \mathrm{~min}$ and then 2 times with $1 \mathrm{X} \mathrm{SSC}, 0.1 \% \mathrm{SDS}$ at $65^{\circ} \mathrm{C}$ for $15 \mathrm{~min}$. Positive bands were developed using a Konica Minolta developer after $5 \mathrm{~h}$ or overnight exposure.

Genomic DNA obtained from transfected and WT cells were amplified by PCR using primers F3001 for upstream of the 5' SSU and A1715 (table 1) designed for the sequence covering downstream of the 5'SSU and upstream of the cpa/cpb/ egfp gene (Fig.1).

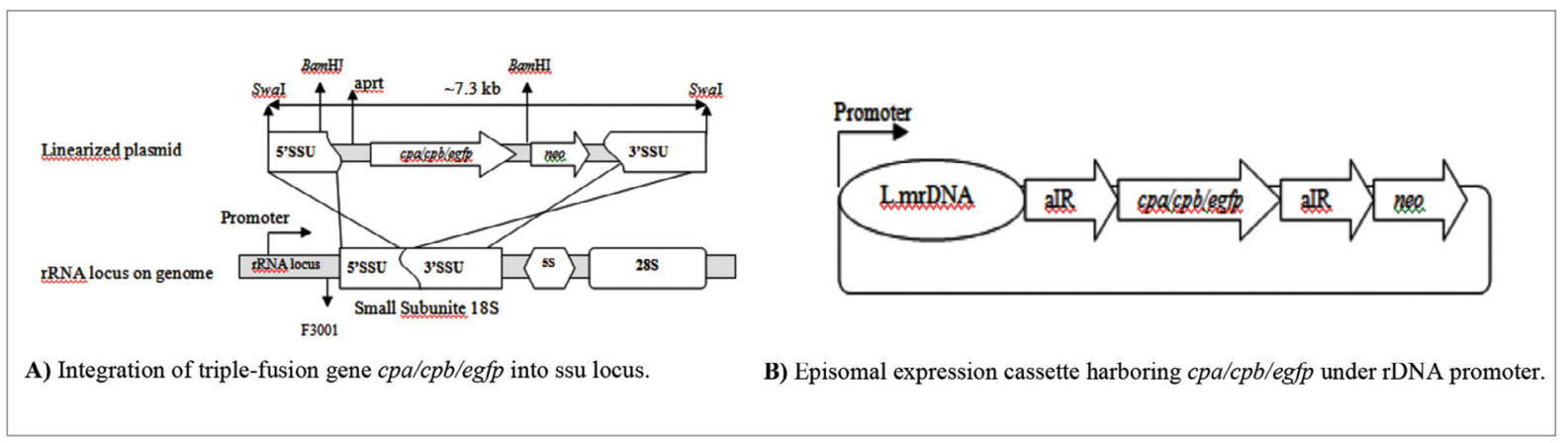

Fig.1. Schematically representation of two expression cassettes.

A) Cassette containing 5'SSU-cpa/cpb/egfp-neo-3'SSU isolated with SwaI for integration in SSU region of L. tarentolae genome. The positions of the promoter, restriction sites and the primers are indicated in the ribosomal locus by black arrowheads. B) Fragment carried by pSP72-L.m rDNA plasmid for episomal expression under the control of rDNA promoter. L.mr DNA: L. major ribosomal DNA, $\alpha$ IR: Intergenic region of the tubulin gene, $c p a / c p b / e g f p$ : fused gene. The direction of transcription is shown by the arrowheads.

Table 1. Sequences of the primers.

\begin{tabular}{|l|l|}
\hline Primers name & Sequences \\
\hline F3001 & 5'-GATCTGGTTGATTCTGCCAGTAG-3' \\
\hline A1715 & 5'-TATTCGTTGTCAGATGGCGCAC-3' \\
\hline H8 & 5'-GACGGATCCGCCCCCAGTGGTGTGATGTCG-3' \\
\hline CPBE & 5'-CTGAAGCTTCACATGCGCGGACACGGG-3' \\
\hline
\end{tabular}

Western blot. Parasites in logarithmic and stationary phase were washed with PBS and solubilized directly in SDS-PAGE sample buffer and separated on $12.5 \%$ acrylamide gels [22]. Separated proteins were transferred onto nitrocellulose membranes (Schleicher \& Schuell, Germany) and blocked overnight at $4{ }^{\circ} \mathrm{C}$. Blots were incubated for $2 \mathrm{~h}$ at room temperature with a 1:6000 dilution of horeseradish peroxidase (HRP)-conjugated anti GFP monoclonal antibody (Acris antibodies $\mathrm{GmbH}$ ) or with a 1:50 dilution of anti-CPB polyclonal antibody from vaccinated mice. HRP-conjugated goat anti-mouse IgG (Sigma, Germany) diluted 1:2500 in blocking solution was used as secondary antibody with anti-CPB. Membranes were developed using DAB as substrate.

RNA extraction and RT-PCR. Total RNA was extracted from 107 parasites in mid-log phase using QIAGEN RNeasy plus Mini Kit (QIAGEN, Germany) according to the manufacturer's instructions and stored at $-80^{\circ} \mathrm{C}$. Approximately, $2 \mu \mathrm{g}$ of total RNA was reverse-transcribed with QIAGEN Omniscript Reverse Transcriptase Kit using oligo dT primers (QIAGEN, Germany). Transcripts of fused $c p a / c p b$ were detected by amplification of the cDNA using primers $\mathrm{H} 8$ and CPBE (Table 1). PCR conditions were one cycle of denaturation at $95^{\circ} \mathrm{C}$ for $5 \mathrm{~min}$ followed by 30 cycles of denaturation at $94^{\circ} \mathrm{C}$ for $1 \mathrm{~min}$, annealing at $62^{\circ} \mathrm{C}$ for $2 \mathrm{~min}$ and extension at $72^{\circ} \mathrm{C}$ for $3 \mathrm{~min}$ and a final extension at $72^{\circ} \mathrm{C}$ for $20 \mathrm{~min}$. Any residual RNA was removed from the synthesized cDNA prior to PCR amplification by treatment of the RT product with RNase (Boehringer, Germany).

Microscopic observation and flow cytometric analysis of parasites. EGFP expression in parasites was monitored by flow cytometry and since episomal transfectant typically show plasmid copy number variation, several clones were examined. Morphology, motility and viability of transfected promastigotes in log and stationary phase were compared with the wild-type by light and Epifluorescence microscopy (Nikon, Japan). As a measure of EGFP expression level in transfected parasites, the fluorescence intensity of at least 105 cells gated out of all events using forward and side scatter values was quantified in GFP channel (FL-1) by flow cytometry (BD FACS Calibur) and the output data was analyzed by FlowJo software version 7.5 (Tree Star Inc., USA). All experiments were repeated at least 3 times.

Fluorescence intensity analysis in vitro. RAW 264.7 cells (Mouse leukaemic monocyte macrophage cell line) were cultured in RPMI medium supplemented with $10 \%$ FBS in 24-well tissue culture plates (Orange, Belgium) at a concentration of $2 \times 105$ cells per well. After $24 \mathrm{~h}$ incubation, media was removed and live promastigotes (transgenic and wild-type) in stationary phase were washed in PBS and added to the cells at multiplicity of infection (MOI) of 10 (ratio of parasite to cell 10:1). After $4 \mathrm{~h}$, wells were washed with PBS to remove the non-phago- 
cytosed parasites around the cells and incubation continued at $37^{\circ} \mathrm{C}$. At different time points $(24,48$ and $72 \mathrm{~h})$ cells were detached by pre-warmed $0.25 \%$ Trypsin-EDTA in PBS and pelleted by centrifugation at $1700 \mathrm{rpm}$ for $7 \mathrm{~min}$. Pellets were resuspended in $1 \mathrm{ml}$ PBS and fluorescence intensity was monitored by flow cytometry and analyzed using FlowJo software. A total of $5 \times 104$ events were acquired at cell gate.

\section{RESULTS}

Plasmid DNA construct containing $c p a / c p b / e g f p$ fusion gene. Expression of the $1644 \mathrm{bp}$ DNA fragment containing cpa/ $c p b$ fusion gene cloned in pCB6 vector and the ability of the hybrid protein to elicit a protective immune response against $L$. major has been shown previously [23]. To examine stable and transient expression of $c p a / c p b$ gene of $L$. major in a homogenous live parasite system for in vitro and in vivo use, a $2.4 \mathrm{~kb}$ cpa/cpb/egfp fragment was introduced in L. tarentolae under the control of the regulatory sequences of rRNA Pol I promoter. For stable transfection and generation of knock-in transgenic parasites, pLEXSY-cpa/cpb/egfp construct containing 5' SSU ( $\sim 860$ bps) and 3' SSU ( 1080 bps) segments homologous to 18S rRNA locus of L. tarentolae was linearized with SwaI, as previously described [19]. SSU sequences are required for integration of fragments flanked by these segments through homologous recombination (Fig. 1A). Clones with integrated $c p a / c p b /$ egfp fused gene were selected on media containing $50 \mu \mathrm{g} / \mathrm{ml}$ of G418 and confirmed by PCR and Southern blot hybridization.

For episomal expression, pSP72-L.m rDNA Pro- $\alpha$ IR- $\alpha$ IR-Neo vector with $L$. major rDNA promoter as well as two intergenic regions of alpha-tubulin ( $\alpha \mathrm{IR})$ gene as guide sequences for proper splicing/polyadenylation of the transcript (Fig. 1B) was used. PCR product of $c p a / c p b / e g f p$ fusion gene was cloned into the vector, flanked on both sides by $\alpha$ IR and cloning was confirmed by PCR, restriction enzyme digestion and sequence analysis.

Transient transfectants harboring pSP72-L.m rDNA-IR-cpa/ cpb/egfp-IR were kept under high concentration of G418 (at least $200 \mu \mathrm{g} / \mathrm{ml}$ ) in liquid media.

Genotype confirmation. Southern blot and PCR were used to confirm the presence of the introduced fusion gene in both integrative and episomal clones (Fig. 2).

For Southern blot hybridization, all genomic DNA were digested with BamHI and a probe covering the egfp gene was used. Positive bands of correct size of $4 \mathrm{~kb}$ and $2.7 \mathrm{~kb}$ for integrated $c p a / c p b / e g f p$ fragment and integrated egfp alone in $L$. tarentolae $e^{e g f p+}$ as positive control were observed [17] (Fig. 2A, lanes 2 and 3). In contrast, the egfp probe did not hybridize to wild-type line even after overnight exposure (Fig. 2A, Lane 1). Furthermore, the presence of the introduced gene and its correct insertion into the parasite genome were verified by PCR using primers listed in table 1 (Fig. 2B). Specific primers of egfp amplified a $\sim 750$ bp product in transfected clones and $L$. tarentolae ${ }^{e g f p+}$ genome as positive control (Fig. 2B, lanes 1 and 4), $\mathrm{H} 8$ and CPBE, primers targeting $c p a / c p b$ sequence, produced a $\sim 1.6 \mathrm{~kb}$ band (Fig. 2B, lane 2) and finally using $\mathrm{H} 8$ and GFPR primers resulted in a $\sim 2.4 \mathrm{~kb}$ product confirming the presence of the triple fused gene in the genome of integrated parasites (Fig. 2B, lane 3). These results suggested that homologous recombination between the two homologue regions has led to the knock-in of triple fused gene sequence into a copy of the rRNA gene.

A further PCR with primers specific for 5' SSU region complementary to genome and vector (F3001 and A1715 Fig. 1) to confirm the insertion of $c p a / c p b / e g f p$ fused gene into the rRNA locus was performed. Integration of $c p a / c p b / e g f p$ by a double crossover event in rRNA locus resulted in a $\sim 1060$ bp PCR product (lane 1 of Fig. 2C).

which confirms the integration of tri-fused gene in $18 \mathrm{~S}$ rRNA locus. Using 5' SSU specific primers on L. tarentolae ${ }^{e g f p+}$ as positive control showed similarly sized product (Fig. 2C, lane 2 ). No amplification product was observed when wild-type genomic DNA was used as a template (Fig. 2C, lane 4).

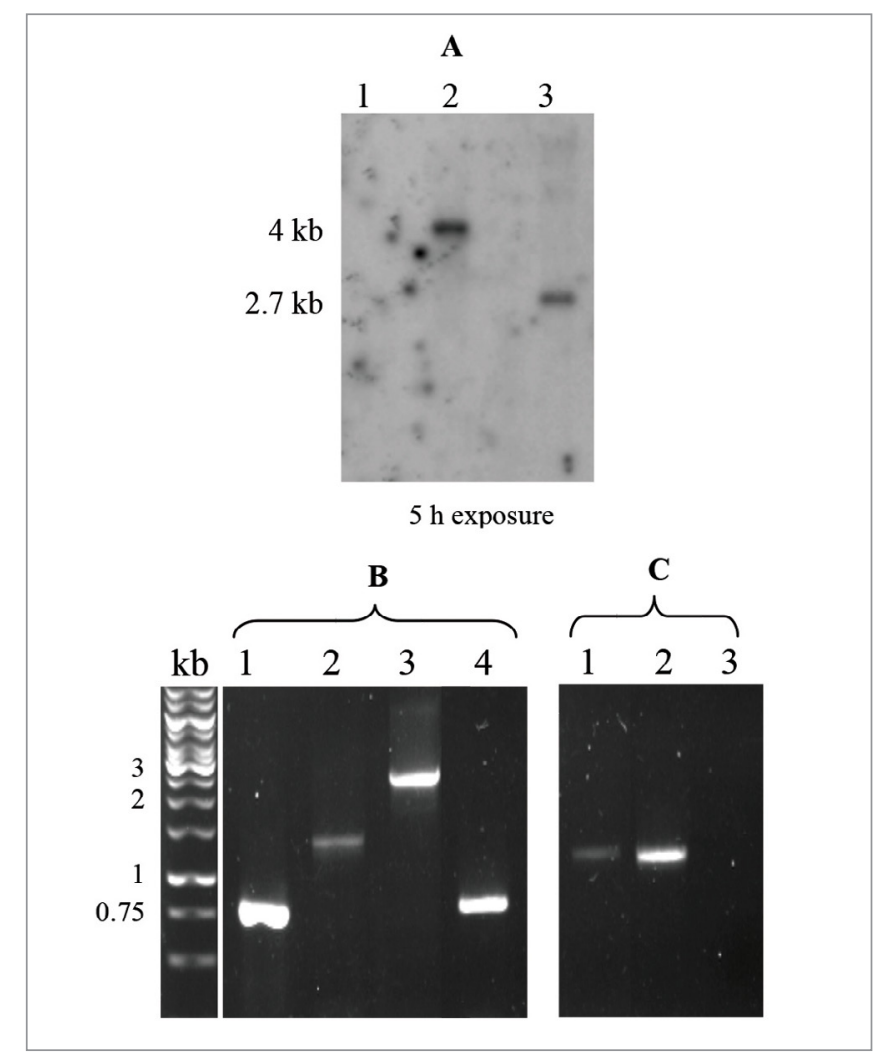

Fig. 2. Presence and integration of the expression cassette into the SSU locus was confirmed by Southern blot and PCR. Genomic DNA was extracted from transgenic and wild-type parasites

A) Southern blot analysis with radiolabeled dCTP. Lane 1: wild-type L. tarentolae; Lane 2: L. tarentolae carrying integrated $c p a / c p b / e g f p$ fused gene; Lane 3: egfp-expressing $L$. tarentolae (L. tarentolae egfp $^{+}$).

B) PCR using different primers showing the presence of triple gene in transiently transfected Leishmania. Lane 1: egfp gene amplified with GFPF/GFPR ( $750 \mathrm{bp}$ ); Lane 2: PCR product of double $c p a / c p b$ fused gene with H8/CPBE primers ( $1300 \mathrm{bp})$; Lane 3: PCR product of triple $c p a / c p b / e g f p$ fused gene with H8/GFPR primers ( 2400 bp): Lane 4: Amplification of egfp gene with

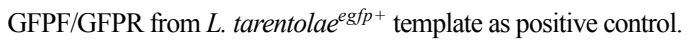

C) The results of PCR to confirm integration of the fused gene in the genome of stably transfected Leishmania. Lane 1: PCR product using specific 5'SSU primers F3001/aprt ( 1 kb) using integrated L. tarentolae $e^{c p a / c p b / e g f p+}$ as template. Lane 2: results of PCR on genome of L. tarentolae ${ }^{e g f p+}$ with 5 'SSU specific primers as positive control. Lane 3: PCR on genome of wild-type $L$. tarentolae with 5 'SSU primers as negative control. 
Analysis of fusion gene expression in transfectants by RT-PCR and Western blot. Transcription of trifused gene at RNA level and its expression were verified using RT-PCR and Western blot, respectively. The amplification of cDNA, synthesized from total RNA of the transgenic and the wildtype parasites in logarithmic phase of growth with $\mathrm{H} 8$ and CPBE designed for $c p a / c p b$ region produced a fragment of $1300 \mathrm{bp}$ from both episomaly - and stably-transfected parasites (Fig. 3A, lanes 2 and 4). RT-PCR with cDNA of wild-type L. tarentolae failed to produce this product (Fig. 3A, Lane 5). Western blot analyses on whole lysates of the integrative and episomal transfectants were performed with two different antibodies, anti-EGFP and anti-CPB. The molecular weight of tri-fused protein (CPA/CPB/EGFP) was estimated at $85 \mathrm{kDa}$ and immunoblot with anti-GFP antibody confirmed expression of the protein in both episomal and integrative transfected parasites (Fig. 3B). Whereas the use of this antibody for L. tarentolae integrated with egfp gene alone produced a $\sim 27$ $\mathrm{kDa}$ band and the wild-type parasites did not react with anti-GFP (Fig. 3B). The chimeric protein was also detectable at both logarithmic and stationary phase of the transfected parasites using anti-CPB antibody (Fig. 3B).

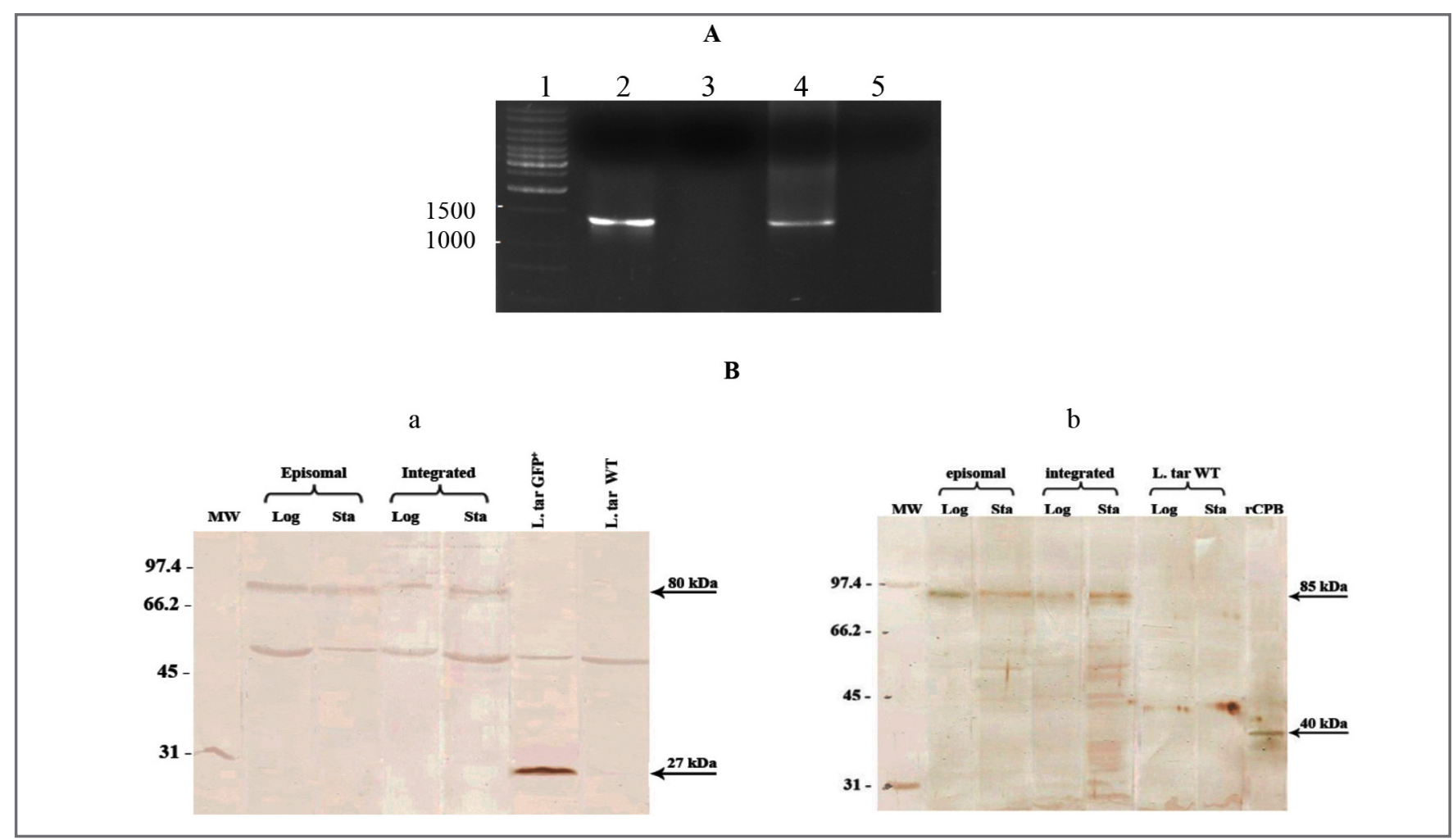

Fig. 3. Comparison of gene expression in parasites by RT-PCR and western blot.

A) RT-PCR of transfected and wild-type (WT) L. tarentolae. Lane 1: DNA molecular weight maker (bp), Lane 2 and 4: amplified cDNA from transfected parasites, lane 3; amplified RNA as negative control, Lane 5: amplified cDNA of wild-type L. tarentolae as negative control. B) Western blot using anti-EGFP and anti-CPB antibodies. a) monoclonal anti-GFP antibody detected a $85 \mathrm{kDa}$

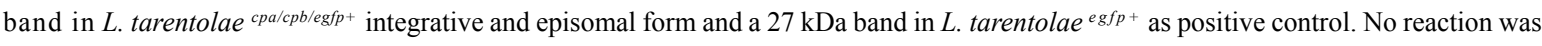
detected in wild-type $L$. tarentolae as negative control. b) Polyclonal anti-CPB antibody detected a $85 \mathrm{kDa}$ band in $L$. tarentolae carrying an episomal construct of $c p a / c p b / e g f p$ gene or integrated into genome in logarithmic and stationary phase. Log: logarithmic phase; Sta: stationary phase; L. tar WT: Leishmania tarentolae wild-type.

Phenotypic characterization of transfected parasites by microscopy and flow cytometry. Morphological features (activity and motility) of the transgenic promastigotes were monitored daily and both L. tarentolae lines carrying cpa/cpb/ egfp genes episomally and integrated, displayed a normal morphology (drop-like shape) of the wild-type strain with a normal length of flagellum.

EGFP expression and intensity were verified and compared by Epifluorescent microscope. EGFP was fused to the CPB C-terminal and its fluorescence signal distributed in the whole cytoplasm. In spite of similarity in morphology of the transfected parasites, fluorescent intensity differed significantly between L. tarentolae $e^{e g f p+}$ and L. tarentolae ${ }^{c p a / c p b / e g f p+}$. No fluorescent signal was detected in the wild-type.

The fluorescent intensity of EGFP in the transfected parasites was measured by flow cytometry. As shown in Table 2, when CPA/
$\mathrm{CPB} / \mathrm{EGFP}$ is expressed stably in L. tarentolae, the green fluorescence intensity was stronger than the episomal form. Increasing the concentration of G418 in the medium did not increase the EGFP expression. Furthermore, the EGFP intensity in the integrated form of the recombinant parasites varied between log and stationary phases with significant decrease in stationary phase. Daily culture of the integrated form of the recombinant parasites in media containing various amounts of G418 showed that $200 \mu \mathrm{g} / \mathrm{ml}$ of G418 increased the fleurescent intensity in the log phasae $(\sim 61.44 \pm 6.12)$, which decreased in higher concentrations $(17.96 \pm 11.26$ for 500 $\mu \mathrm{g} / \mathrm{ml}$ ). Increasing the concentration of G418 to more than $500 \mu \mathrm{g} /$ $\mathrm{ml}$ had no effect on EGFP intensity (data not shown).

This association between the increase in G418 and EGFP intensity was also observed in stationary phase of the parasites, cultured daily in the presence of varying amounts of G418. Expression of this gene in L. tarentolae egfp+ $^{\text {was much higher }}$ 
when expressed alone compred to the fused form, was stable $(>\sim 97 \%)$ and independent of growth phase and G418 concentration (Table 2).

Confirmation of fusion gene expression by COS-7 Cells. Expression of the EGFP-tagged fusion gene was also confirmed using COS-7 cells transfected with pEGFP-cpa/cpb/ egfp recombinant vector or pEGFP alone by PEI $25 \mathrm{kDa}$ as a transfection reagent. Comparison of EGFP fluorescent intensity in transfected cells with pEGFP-cpa/cpb/egfp vector with pEGFP bearing cells by fluorescence microscopy showed the expression of GFP in both groups, but the intensity was higher in cells transfected with pEGFP containing egfp gene alone (Fig. 4B). The flow cytometry results indicated that the level of intensity at $24 \mathrm{~h},(35.32 \%)$ decreased to $15.79 \%$ at $48 \mathrm{~h}$ and $12.58 \%$ at $72 \mathrm{~h}$.

Table 2. Expression of the egfp gene in different growth phases of the parasite with different concentrations of G418 by FACS analysis. Data is shown as the mean \pm SD from 3 independent experiments

\begin{tabular}{|c|c|c|c|}
\hline Expression condition & {$[\mathbf{G 4 1 8}] \mu \mathrm{g} / \mathrm{ml}$} & $\begin{array}{c}\text { Log } \\
(\% \text { intensity) }\end{array}$ & $\begin{array}{c}\text { Stationary } \\
\text { (\% intensity) }\end{array}$ \\
\hline \multirow{3}{*}{ L. tarentola $e^{c p a / c p b / e g f p^{+}}$(integrative) } & - & $0.4 \pm 0.24$ & - \\
\hline & 200 & $61.44 \pm 6.12$ & $4.88 \pm 2.6$ \\
\hline & 500 & $17.96 \pm 11.26$ & $9.94 \pm 0.95$ \\
\hline L. tarentolae ${ }^{c p a / c p b / e g f p+}$ (episomally) & 500 & $49.17 \pm 9.8$ & $21.54 \pm 1.62$ \\
\hline L. tarentolae $e^{\text {egfp+ }}$ & $0-500$ & $98.76 \pm 0.8$ & $98.3 \pm 0.5$ \\
\hline L. tarentolae (Wild-Type) & - & 0.35 & - \\
\hline
\end{tabular}

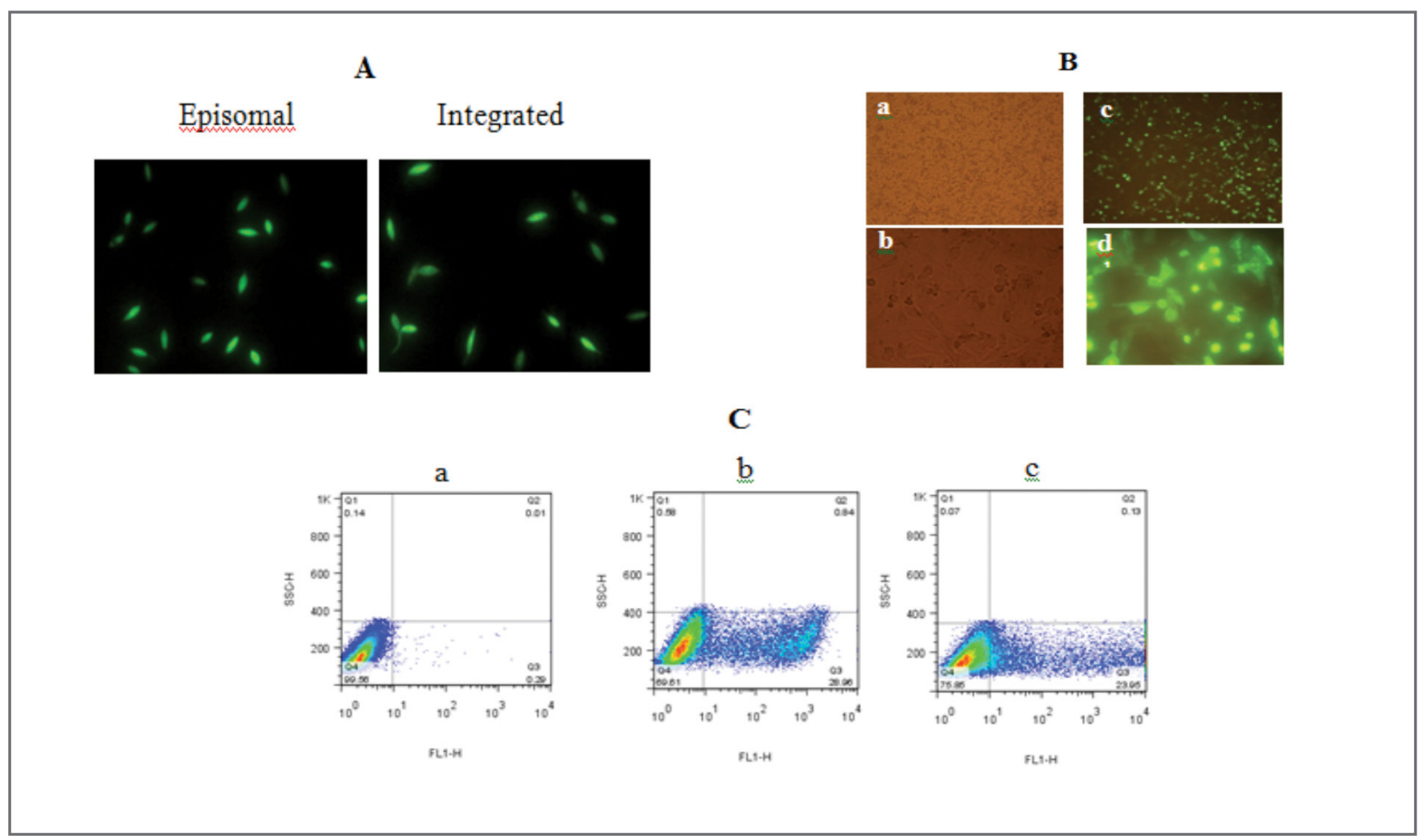

Fig. 4. Fluorescence microscopy and flow cytometry results of episomal and integrative fusion gene expression in COS-7 cells A) L. tarentolae integrated with $c p a / c p b / e g f p$ and L. tarentolae carrying an episomal gene expressing cpa/cpb/egfp grown in $200 \mu \mathrm{g} /$ $\mathrm{ml}$ of G418. B) Fluorescent microscopy of infected COS-7. a) Uninfected cells; b) cells infected with wild-type L. tarentolae; c) cells infected with $L$. tarentolae integrated with $c p a / c p b / e g f p$; d) cells infected with L. tarentolae ${ }^{e g f p+}$. C) Flow cytometry analysis of infected COS-7 cells at $48 \mathrm{~h}$ after infection. a) Non-infected COS-7; b) Infected COS-7 with $c p a / c p b / e g f p$ gene (pEGFP-cpa/cpb/egfp), c) Infected COS-7 with egfp gene ( $\mathrm{pEGFP}$ ).

In vitro infection with integrated form of $L$. tarentola $^{c p a / c p b / e g f p^{+}}$. The intensity of EGFP was estimated in intracellular form of the transfected Leishmania by FACS-based screening assays, fluorescence microscopy and RT-PCR in RAW 264.7 cell line. The infected cells were assessed at different time points of 24 , 48 and $72 \mathrm{~h}$ post-infection via FACS analysis. The EGFP pos- itive cells were representative of cell population infected with L. tarentolae integrated with $c p a / c p b / e g f p$ or egfp alone as the positive control. The percentages obtained were gated based on infected cells with recombinant $L$. tarentolae expressing EGFP. The best time to survey the intracellular form was $48 \mathrm{~h}$ post-infection, since $24 \mathrm{~h}$ was not enough for the parasites to differ- 
entiate and after $72 \mathrm{~h}$, a significant decrease in the infection rate was observed, due to the lysis of many cells caused by the infection (Table 3).

At $48 \mathrm{~h}$ post infection, total RNA was extracted from the infected cells with transgenic and WT parasites and were re- verse transcribed to cDNA. Subsequently, PCR using H8 and CPBE primers amplified a $1300 \mathrm{bp}$ fragment of $c p a / c p b$ fused gene confirming the transcription of fused $c p a / c p b$ genes (Fig. 5). The negative controls showed no background or DNA contamination.

Table 3. Percentage of EGFP positive cells in infected RAW 264.7 cell lines with $L$. tarentolae integrated with $c p a / c p b / e g f p$ by flow cytometry at 24,48 and $72 \mathrm{~h}$ post-infection

\begin{tabular}{|c|c|c|c|}
\hline Expression condition & $\mathbf{2 4} \mathbf{~ h}$ & $\mathbf{4 8} \mathbf{~ h}$ & $\mathbf{7 2} \mathbf{~ h}$ \\
\hline RAW + L. tarentolae & cpa/cpb/egfp ${ }^{+}$ & 19.41 & 1.32 \\
\hline RAW+L. tarentolae $e^{e g f p^{+}}$ & 21.49 & 33.23 & 20.25 \\
\hline L. tarentolae (Wild-Type) & 29.14 & 1.01 & 1.30 \\
\hline RAW & 1.47 & 0.32 & 0.02 \\
\hline
\end{tabular}

A

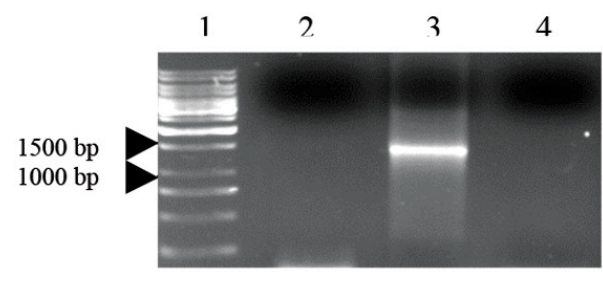

B

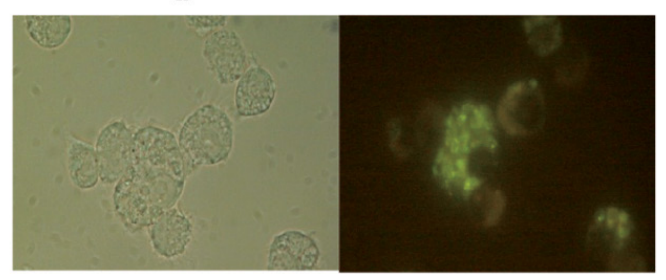

C

a

b

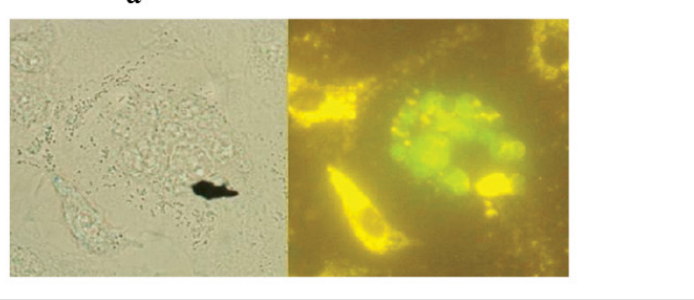

Fig. 5. RT-PCR and microscopic results of RAW 264.7 cell line infected with $L$. tarentolae integrated with $c p a / c p b / e g f p$

A) RT-PCR results of RAW 264.7 cell line, $48 \mathrm{~h}$ after infection with L. tarentolae integrated with $c p a / c p b / e g f p$ using H8 CPBE primers. Lane 1, MW maker, Lane 2 and 3, amplification of the extracted RNA, and cDNA from L. tarentolae cpa/cpb/egfp+ $^{\text {inte- }}$ gratively infected RAW 264.7, Lane 4, no template PCR control. B) RAW 264.7 cell line infected with $L$. tarentolae integrated with $c p a / c p b / e g f p$; a) Light microscope. b) Fluorescent microscope. C) Infected cell line with $L$. tarentolae tgfp+ $^{\text {as a }}$ positive control. a) Light microscopy. b) Fluorescent microscopy

\section{DISCUSSION}

Several reports have suggested that recombinant $L$. tarentolae could be an ideal system to express proteins in native form for vaccination [24-25]. In principle, unlike classical prokaryotic systems, Leishmania contains all the required components for proper folding of proteins and post-translational modifications. The main advantage of this system is production of proteins in native form without the need for extra processing. One of the differences between Leishmania and prokaryotes is the regulation of protein expression through intergenic untranslated regions (UTRs) [26]. The potential of $L$. tarentolae in expressing heterologous proteins has been shown previously [24]. The hetero-trimeric recombinant LM-332 (Laminin) containing three $\alpha, \beta$ and $\gamma$ chains has been expressed and purified successfully from L. tarentolae after sequential stable transfection of three different constructs. The LM protein is a high molecular weight glycoprotein, rich in cysteine residues that requires proper folding and was secreted into the media as an active protein with correct folding due to the function of endogenous chaperon proteins of the host parasite [27].

Recombinant proteins can be expressed in Leishmania transiently or stably and to select the best approach for expression of $c p a / c p b / e g f p$ chimeric gene, the episomal and integrative transfections were compared. Therefore, the triple fusion gene was integrated into the rRNA locus downstream of $18 \mathrm{~S}$ ribosomal promoter [19] to be transcribed by RNA Pol I, or expressed episomally under regulation signals or regulatory elements of RNA Pol I. However, in both systems, exogenous protein was expressed from the strong rRNA promoter and transcribed by the endogenous RNA Pol I $[18,28]$. The specific sequences of ribosomal rRNA promoter in plasmid vector allowed replication and its stable maintenance during inheritance as a single copy [29].

Recently, we used successfully recombinant $L$. tarentolae expressing triple fused genes including $L$. donovani A2 antigen along with cysteine proteinases (CPA and CPB without its unusual $\mathrm{C}$-terminal extension (CPB-CTE)) as a live vaccine [8].

Here, we evaluated the expression potential of triple-fusion (CPA/CPB/EGFP) protein and EGFP as a reporter in 
two recombinant parasite lines. Various methods were used to verify the insertion of the tri-fused gene integratively and its expression in both episomal and stably transfected parasites, but the results pertaining to COS-7 cells infection showed that the expression of EGFP protein alone in L. tarentolae was higher compared to the fusion protein.

The rRNA gene of Leishmania which is able to induce constitutive and high level expression of exogenous integrated genes are located in multiple loci and are tandemly repeated in variable numbers. Therefore to increase the expression of heterologous genes in this host, the use of multiple integrations in rRNA locus by repeated transfection with a linear construct has been suggested [30]. Expression of GFP as measured by Fluorescent intensity is highly dependent on various factors such as, the number of the parasites multiplied in different phases, the copy number of the integrated gene or the plasmid construct, the chaperones, as well as size, solubility, charge and folding of the fusion protein [31].

It has been shown that the higher level of metabolism in parasites during the logarithmic phase resulted in higher levels of gene expression [32]. Our data also showed that in the $\log$ phase, the intensity of EGFP was proportional to amplification of the parasites and was 2-12 times higher than the stationary phase. Expression level of EGFP alone was higher than when tagged to $\mathrm{CPA} / \mathrm{CPB}$ which could be due to the fact that EGFP is a small protein $(27 \mathrm{kDa})$, and it is easier for the host to express it, compared to the fusion protein with a longer sequence and a higher molecular weight [33].

The fusion protein was constitutively expressed in the cytoplasm without the need for any selective pressure such as G418 which is beneficial for in vivo applications such as vaccinations; however, in case of toxic gene products, the constitutive expression of foreign genes in the host would not be possible. Compared to transient expression, stable transfection is time consuming but the disadvantage of expression by plasmid vectors is the variability in the number of the transfected parasites and the instability of the vectors in the absence of the drug pressure [34].

In conclusion, $\mathrm{CPA} / \mathrm{CPB} / \mathrm{EGFP}$ fusion protein was successfully expressed transiently in $L$. tarentolae using episomal vectors and this mode of expression is a convenient method for over expression of genes of interest in a short period of time. Furthermore, this study indicated that the L. tarentolae is a suitable eukaryotic host to produce eukaryotic proteins which depending on the objective of the study, both episomal and integrated approaches could successfully be applied.

\section{ACKNOWLEDGEMENTS}

We would like to acknowledge the help of Dr. Ali Eslamifar (Department of Clinical Research, Pasteur institute of Iran) for flow cytometry facility and Hiva Azizi (Laval University, Quebec, Canada,) for performing the Southern blot. We also thank Dr. Amir Mizbani (University Hospital Zurich, Switzerland) for English editing and S. Alizadeh (Molecular Immunology and Vaccine Research Lab, Pasteur institute of
Iran) for his technical assistance.

\section{CONFLICT OF INTEREST}

The authors declare that they have no competing interests.

\section{REFERENCES}

1. Alvar J, Velez I D, Bern C, Herrero M, Desjeux P, Cano J, Jannin J, den Boer M. Leishmaniasis Worldwide and Global Estimates of Its Incidence. PLoS One, 2012; 7 (5): e 35671.

2. Gupta S, Nishi. Visceral leishmaniasis: experimental models for drug discovery. Indian J Med Res, 2011; 133: 27-39.

3. Sahdev S, Khattar SK, Saini KS. Production of active eukaryotic proteins through bacterial expression systems: a review of the existing biotechnology strategies. Mol Cell Biochem, 2008; 307 (1-2): 249-64. 4. Fernandez-Robledo JA, Vasta GR. Production of recombinant proteins from protozoan parasites. Trends Parasitol, 2010; 26 (5): 244-54 5. Silvestre R, Cordeiro-da-Silva A, Ouaissi A. Live attenuated Leishmania vaccines: a potential strategic alternative. Arch Immunol Ther Exp (Warsz), 2008; 56 (2): 123-6.

6. Taylor VM, Muñoz DL, Cedeño DL, Vélez ID, Jones MA, Robledo SM. Leishmania tarentolae: utility as an in vitro model for screening of antileishmanial agents. Exp Parasitol, 2010; 126 (4): 471-5.

7. Niimi T. Recombinant protein production in the eukaryotic protozoan parasite Leishmania tarentolae: a review. Methods Mol Biol, 2012; 824: 307-15.

8. Saljoughian N, Taheri T, Zahedifard F, Taslimi Y, Doustdari F, Bolhassani A, Doroud D, Azizi H, Heidari K, Vasei M, Namvar Asl N, Papadopoulou B, et al. Development of Novel Prime-Boost Strategies Based on a Tri-Gene Fusion Recombinant L. tarentolae Vaccine against Experimental Murine Visceral Leishmaniasis. PLoS Negl Trop Dis, 2013; 7 (4): e2174.

9. Pollock KG, McNeil KS, Mottram JC, Lyons RE, Brewer JM, Scott P, Coombs GH, Alexander J. The Leishmania mexicana cysteine protease, CPB2.8, induces potent Th2 responses. J Immunol, 2003; 170 (4): 1746-53.

10. Mottram JC, Coombs GH, Alexander J. Cysteine peptidases as virulence factors of Leishmania. Curr Opin Microbiol, 2004; 7 (4): 375-81. 11. Silva-Almeida M, Pereira BA, Ribeiro-Guimarães ML, Alves CR. Proteinases as virulence factors in Leishmania spp. infection in mammals. Parasit Vectors, 2012; 5: 160.

12. Rafati S, Fasel N, Masina S. Leishmania cysteine proteinases: from gene to subunit vaccine. Current Genomics, 2003; 4: 253-61.

13. Saravia NG, Escorcia B, Osorio Y, Valderrama L, Brooks D, Arteaga L, Coombs G, Mottram J, Travi BL. Pathogenicity and protective immunogenicity of cysteine proteinase-deficient mutants of Leishmania mexicana in non-murine models. Vaccine, 2006; 24 (19): 4247-59.

14. Dube A, Gupta R, Singh N. Reporter genes facilitating discovery of drugs targeting protozoan parasites. Trends Parasitol, 2009; 25 (9): 432-9.

15. Singh N, Gupta R, Jaiswal AK, Sundar S, Dube A. Transgenic Leishmania donovani clinical isolates expressing green fluorescent protein constitutively for rapid and reliable ex vivo drug screening. J Antimicrob Chemother, 2009; 64 (2): 370-4.

16. Mehta SR, Huang R, Yang M, Zhang XQ, Kolli B, Chang KP, Hoffman RM, Goto Y, Badaro R, Schooley RT. Real-time in vivo green fluorescent protein imaging of a murine leishmaniasis model as a new tool for Leishmania vaccine and drug discovery. Clin Vaccine Immunol, 2008; 15 (12): 1764-70. 
17. Bolhassani A, Taheri T, Taslimi Y, Zamanilui S, Zahedifard F, Seyed N, Torkashvand F, Vaziri B, Rafati S. Fluorescent Leishmania species: development of stable GFP expression and its application for in vitro and in vivo studies. Exp Parasitol, 2011; 127 (3): 637-45.

18. Misslitz A, Mottram JC, Overath P, Aebischer T. Targeted integration into a rRNA locus results in uniform and high level expression of transgenes in Leishmania amastigotes. Mol Biochem Parasitol, 2000; 107(2): 251-61.

19. Zahedifard F, Gholami E, Taheri T, Taslimi Y, Doustdari F, Seyed N, Torkashvand F, Meneses C, Papadopoulou B, Kamhawi S, Valenzuela JG, Rafati. Enhanced Protective Efficacy of Nonpathogenic Recombinant Leishmania tarentolae Expressing Cysteine Proteinases Combined with a Sand Fly Salivary Antigen. PLoS Negl Trop Dis, 2014; 8 (3): e2751.

20. Abdian N, Gholami E, Zahedifard F, Safaee N, Rafati S. Evaluation of DNA/DNA and prime-boost vaccination using LPG3 against Leishmania major infection in susceptible BALB/c mice and its antigenic properties in human leishmaniasis. Exp Parasitol, 2011; 127 (3): 627-36.

21. Robinson KA. Beverley SM. Improvements in transfection efficiency and tests of RNA interference (RNAi) approaches in the protozoan parasite Leishmania. Mol Biochem Parasitol, 2003; 128 (2): 217-28.

22. Mizbani A, Taheri T, Zahedifard F, Taslimi Y, Azizi H, Azadmanesh K, Papadopoulou B, Rafati S. Recombinant Leishmania tarentolae expressing the A2 virulence gene as a novel candidate vaccine against visceral leishmaniasis. Vaccine, 2009; 28 (1): 53-62.

23. Zadeh-Vakili A, Taheri T, Taslimi Y, Doustdari F, Salmanian AH, Rafati S. Immunization with the hybrid protein vaccine, consisting of Leishmania major cysteine proteinases Type I (CPB) and Type II (CPA), partially protects against leishmaniasis. Vaccine, 2004; 22 (1516): $1930-40$.

24. Kovtun O, Mureev S, Johnston W, Alexandrov K. Towards the construction of expressed proteomes using a Leishmania tarentolae based cell-free expression system. PLoS One, 2010; 5 (12): e14388.

25. Mizbani A, Taheri T, Zahedifard F, Taslimi Y, Azizi H, Azadmanesh K, Papadopoulou B, Rafati S. Recombinant Leishmania tarentolae expressing the A2 virulence gene as a novel candidate vaccine against visceral leishmaniasis. Vaccine, 2009; 28 (1): 53-62.

26. Clayton C, Shapira M. Post-transcriptional regulation of gene expression in trypanosomes and leishmanias. Mol Biochem Parasitol, 2007; 156 (2): 93-101.

27. Phan HP, Sugino M, Niimi T. The production of recombinant human laminin-332 in a Leishmania tarentolae expression system. Protein Expr Purif, 2009; 68 (1): 79-84.

28. Orlando TC, Mayer MG, Campbell DA, Sturm NR, Floeter-Winter LM. RNA polymerase I promoter and splice acceptor site recognition affect gene expression in non-pathogenic Leishmania species. Mem Inst Oswaldo Cruz, 2007; 102 (7): 891-4.

29. Boucher N, McNicoll F, Laverdière M, Rochette A, Chou MN, Papadopoulou B. The ribosomal RNA gene promoter and adjacent cis-acting DNA sequences govern plasmid DNA partitioning and stable inheritance in the parasitic protozoan Leishmania. Nucleic Acids Res, 2004; 32 (9): 2925-36.

30. Kushnir S, Cirstea IC, Basiliya L, Lupilova N, Breitling R, Alexandrov K. Artificial linear episome-based protein expression system for protozoon Leishmania tarentolae. Mol Biochem Parasitol, 2011; 176 (2): 69-79.

31. Kim CW, Han KS, Ryu KS, Kim BH, Kim KH, Choi SI, Seong BL. $\mathrm{N}$-terminal domains of native multidomain proteins have the potential to assist de novo folding of their downstream domains in vivo by acting as solubility enhancers. Protein Sci, 2007; 16 (4): 635-43.
32. Lang T, Goyard S, Lebastard M, Milon G. Bioluminescent Leishmania expressing luciferase for rapid and high throughput screening of drugs acting on amastigote-harbouring macrophages and for quantitative real-time monitoring of parasitism features in living mice. Cell Microbiol, 2005; 7 (3): 383-92.

33. Pedelacq JD, Cabantous S, Tran T, Terwilliger TC, Waldo GS. Engineering and characterization of a superfolder green fluorescent protein. Nat Biotechnol, 2006; 24 (1): 79-88.

34. Roy G, Dumas C, Sereno D, Wu Y, Singh AK, Tremblay MJ, Ouellette M, Olivier M, Papadopoulou B. Episomal and stable expression of the luciferase reporter gene for quantifying Leishmania spp. infections in macrophages and in animal models. Mol Biochem Parasitol, 2000; 110 (2): 195-206. 